\title{
Two-point measurement of amylase in drainage fluid predicts severe postoperative pancreatic fistula after gastric cancer surgery
}

\author{
Satoshi Kamiya ${ }^{1} \cdot$ Naoki Hiki $^{1} \cdot$ Koshi Kumagai $^{1} \cdot$ Michitaka Honda $^{1} \cdot$ Souya Nunobe ${ }^{1} \cdot$ Manabu Ohashi $^{1}$. \\ Takeshi Sano ${ }^{1} \cdot$ Toshiharu Yamaguchi $^{1}$
}

Received: 30 November 2017 / Accepted: 27 January 2018 / Published online: 13 February 2018

(c) The International Gastric Cancer Association and The Japanese Gastric Cancer Association 2018

\begin{abstract}
Background Early identification of patients at risk of postoperative pancreatic fistula (POPF) allows appropriate management after gastrectomy. Although some reports have suggested a correlation between POPF and the concentration of amylase in drained abdominal fluid (D-AMY), this has not been proven to impact sufficiently on clinical decision-making. A sustained high level of D-AMY is often assumed to be due to unsatisfactory drainage or excessive pancreatic leakage. We assessed the clinical utility of measuring D-AMY on postoperative day (POD) 1 and POD3 for prediction of POPF.

Methods Starting in April 2014, 801 patients who underwent radical gastrectomy with prophylactic drain placement were consecutively enrolled. We routinely measured D-AMY on POD1 and POD3, and compared the incidence of problematic POPF and clinical factors including D-AMY. We also attempted to clarify whether such two-point D-AMY measurement was clinically useful for patient management after gastrectomy.

Results Fifty-one of the patients (6.4\%) developed Clavien-Dindo grade III or worse POPF. Using D-AMY cutoffs of 2218 IU/L on POD1 and 555 IU/L on POD3, the patients were successfully classified. The highest risk group, in which D-AMY was higher than the cut-off value on both POD1 and POD3, showed a significantly high rate of occurrence (33/105, $31.4 \%$ ) and high positive likelihood ratio (6.74). Multivariate analysis showed that classification into this highest risk group was an independent risk factor for development of severe POPF (odds ratio 15.2, 95\% CI 7.92-29.0).

Conclusion Two-point measurement of D-AMY may be an efficient tool for achieving individualized management of POPF following radical gastrectomy.
\end{abstract}

Keywords Gastric cancer · Gastrectomy · Amylase · Complication · Pancreatic fistula

\section{Introduction}

In patients undergoing gastric cancer surgery, intraperitoneal bleeding and abdominal abscess caused by postoperative pancreatic fistula (POPF) and pancreatic leakage are the most potentially serious and life-threatening postoperative complications. Although these complications are usually treated after the levels of inflammation markers have increased or an abscess has formed, accurate prediction of severe POPF in the early postoperative period would provide a chance to manage affected patients in advance. If POPF

Naoki Hiki

naoki.hiki@jfcr.or.jp

1 Division of Surgery, Cancer Institute Hospital of Japanese Foundation for Cancer Research, 3-8-31, Ariake, Koto, Tokyo 135-8550, Japan could be accurately predicted, abscess formation and severe infection might be preventable by means of early therapeutic interventions such as administration of appropriate antibiotics and drainage of excess fluid. To detect the potential for POPF, the amylase concentration in drained abdominal fluid (D-AMY) taken through an indwelling intra-abdominal drain is often measured in clinical practice. Several articles have reported a close relationship between POPF and D-AMY [1-8]. However, the cut-off value of D-AMY for prediction of POPF and the timing of D-AMY measurement have differed, and the accuracy of this parameter is sometimes insufficient for treatment decision-making [1-11].

Theoretically, D-AMY can directly and objectively indicate the amount of pancreatic leakage and the risk of consequent intra-abdominal abscess, regardless of the preoperative patient's condition, type of surgical procedure, and also surgeon's skill [12]. Clinically, a continuously maintained 
high D-AMY level might suggest persistent pancreatic leakage or insufficient drainage, which could result in clinically problematic POPF. The significance of multiple measurements of D-AMY to improve diagnostic accuracy, for example, by taking samples on different days, is still unclear.

In the present study, we hypothesized that a continuously high D-AMY level after radical gastrectomy would predict the development of clinically problematic POPF. We collected D-AMY samples on POD1 and POD3 and assessed the clinical impact of this two-point measurement for more accurate prediction of POPF.

\section{Materials and methods}

\section{Patients}

The subjects of this study were consecutive patients with gastric cancer who underwent gastrectomy with more than D1 plus lymph node dissection in the Cancer Institute Hospital of the Japanese Foundation for Cancer Research between April 2014 and December 2015. As this study was intended to evaluate the predictability of D-AMY after standard gastrectomy, we excluded patients with remnant stomach cancer, patients who underwent thoracotomy, and patients who underwent combined resection due to the involvement of other organs, with the exception of only cholecystectomy and splenectomy. The protocol of this study was approved by the Institutional Review Board of the Cancer Institute Hospital. This report had been written referring STRAD statement.

\section{Surgical procedures}

The patients' clinical stages were determined according to the Japanese Classification of Gastric Cancer, 3rd English edition [13] and the UICC TNM classification, 7th edition [14]. Surgical procedures were performed according to the Japanese Gastric Cancer Treatment Guidelines 2014 (ver. 4) [15] and six senior surgeons specializing in gastric cancer surgery were responsible for all operations. Laparoscopic gastrectomy was performed with D1 plus lymph node dissection for patients with clinically diagnosed T1N0M0 cancer, and with D2 lymph node dissection for those with T2N0M0 or T1N1M0 cancer. Surgery with laparotomy was adopted for patients with more advanced cancer. At the close of the operation, the abdominal cavity was washed with warm saline: $1 \mathrm{~L}$ for patients who underwent laparoscopy and $3 \mathrm{~L}$ for those who underwent open surgery. A portable negative pressure drain was routinely placed near the upper border of the pancreas for drainage of excess fluid. During the study period, we routinely took samples of drained fluid through the drainage tube in the early morning on POD1
Table 1 Patient characteristics

\begin{tabular}{|c|c|}
\hline Clinical characteristics & Value \\
\hline \multicolumn{2}{|l|}{ Sex } \\
\hline Male, $n(\%)$ & $507(63)$ \\
\hline Female, $n(\%)$ & $294(37)$ \\
\hline \multicolumn{2}{|l|}{ Age in years } \\
\hline Median (range) & $66(24-98)$ \\
\hline \multicolumn{2}{|l|}{ Body mass index in $\mathrm{kg} / \mathrm{m}^{2}$} \\
\hline Median (range) & $22.8(15.2-39.5)$ \\
\hline \multicolumn{2}{|l|}{ Tumor location } \\
\hline Upper third, $n(\%)$ & $200(25)$ \\
\hline Middle third, $n(\%)$ & $423(53)$ \\
\hline Lower third, $n(\%)$ & $178(22)$ \\
\hline \multicolumn{2}{|l|}{ cT category } \\
\hline $1 / 2 / 3 / 4, n$ & $471 / 124 / 32 / 174$ \\
\hline \multicolumn{2}{|l|}{$\mathrm{cN}$ category } \\
\hline $0 / 1 / 2 / 3, n$ & $654 / 109 / 34 / 4$ \\
\hline \multicolumn{2}{|l|}{ cM category } \\
\hline $0 / 1, n$ & $791 / 10$ \\
\hline \multicolumn{2}{|l|}{ cStage } \\
\hline I/II/III/IV, $n$ & $572 / 120 / 99 / 10$ \\
\hline \multicolumn{2}{|l|}{ Approach } \\
\hline Open, $n(\%)$ & $269(34)$ \\
\hline Laparoscopy, $n(\%)$ & $532(66)$ \\
\hline \multicolumn{2}{|l|}{ Procedure } \\
\hline DG, $n(\%)$ & $458(57)$ \\
\hline PG, $n(\%)$ & $59(7)$ \\
\hline TG, $n(\%)$ & $143(18)$ \\
\hline PPG, $n(\%)$ & $141(18)$ \\
\hline \multicolumn{2}{|l|}{ Surgical duration in min } \\
\hline Median (range) & $295(115-605)$ \\
\hline \multicolumn{2}{|l|}{ Blood loss in $g$} \\
\hline Median (range) & $70(0-2700)$ \\
\hline \multicolumn{2}{|l|}{ D-AMY in IU/L } \\
\hline POD1, median (range) & $925.5(34-34024)$ \\
\hline POD3, median (range) & $267(11-40564)$ \\
\hline
\end{tabular}

$D-A M Y$ amylase concentration of drained fluid, $D G$ distal gastrectomy, $P G$ proximal gastrectomy, $P O D$ postoperative day, $P P G$ pylorus preserving gastrectomy, $T G$ total gastrectomy

and POD3 and measured the D-AMY concentration. When more than two drains were placed, we adopted the highest D-AMY concentration for this analysis.

\section{Definition of POPFs and treatments}

The diagnostic criteria for POPF have not been uniformly defined in the field of gastric cancer surgery, and instead the criteria for pancreatic surgery, International Study Group on Pancreatic Fistula (ISGPF) criteria, have been applied after gastrectomy $[16,17]$. In this study, we aimed to evaluate the 
ability of D-AMY to predict clinically problematic POPF. We adopted the Clavien-Dindo (CD) classification for POPF grading [18] and considered grade III or higher POPF to be clinically problematic, requiring some sort of invasive intervention to treat it. This is equivalent to ISGPF grade B or $\mathrm{C}$, although patients treated with antibiotics alone were excluded considering the intensity of treatment. Every case of POPF was graded at the time of discharge. Measured D-AMY was not used for assessment of POPF in this study. Any inflammation or abscess obviously caused by anastomotic leakage or bleeding was not diagnosed as POPF. Prophylactic antibiotics were administered to all patients from the start of the operation until the following morning, and therapeutic antibiotics were additionally given when infectious complications were suspected due to the presence of a high fever, elevation of inflammatory markers and specific symptoms. For patients who had intense inflammation and were suspected to have abdominal abscess or insufficient drainage, the drainage tube was exchanged and adjusted its place under X-ray guidance to keep an optimal drainage. During this examination, we passed a contrast agent through the catheter to clarify the intra-abdominal abscess cavity or other fistulas. In addition, we continued to monitor the appearance of drained fluid and replaced the drainage tube at intervals of a few days to prevent tube clogging for the cases with clinical suspicion of POPF.

\section{Statistical analysis}

In this study, qualitative variables were compared using Fisher's exact test and Chi-squared test, and quantitative variables were analyzed by Mann-Whitney $U$ test for unequal variables. Optimal D-AMY cut-off levels for prediction of POPF were calculated by deriving the receiver operating characteristic (ROC) curve, and the incidence of POPF was compared with diagnostic accuracy using the D-AMY level. Diagnostic accuracy was also compared using the likelihood ratio (LR) and diagnostic odds ratio (DOR), which are not affected by complication rate, to improve the applicability of our results. LRs may range from 0 to infinity. LRs greater than 1 argue for the diagnosis of interest; the bigger the number, the more convincingly the finding suggests that disease. LRs between 0 and 1 argue against the diagnosis of interest; the closer the LR is to 0 , the less likely the disease. LRs equal to 1 lack diagnostic value. DOR is the ratio of the odds of positivity in subjects with disease relative to the odds in subjects without disease. The relationship between the incidence of POPF and clinical factors including D-AMY on POD1 and POD3 was evaluated by univariate and multivariate analyses. Differences were considered significant if the $P$ value from a two-sided test was $\leq 0.05$. All statistical analyses were performed with EZR (Saitama Medical Center, Jichi Medical University, Saitama, Japan), a graphical user interface for R (The R Foundation for Statistical Computing, Vienna, Austria):

(Positive/negative) likelihood ratio (LR)

$=\frac{\text { probability of (positive/negative) finding in patients with disease }}{\text { probability of (positive/negative) finding in patients without disease }}$

Positive likelihood ratio $(\operatorname{LR}(+))=$ sensitivity/(1-specificity)

Negative likelihood ratio $(\operatorname{LR}(-))=(1-$ sensitivity $) /$ specificity

Diagnostic odds ratio (DOR)

$=$ positive likelihood ratio/negative likelihood ratio.

\section{Results}

\section{Patient characteristics}

A total of 868 patients underwent gastrectomy with lymph node dissection. Thirteen patients who underwent gastrectomy with combined resection, 18 who underwent simultaneous surgery of other organs, and 36 who did not undergo measurement of D-AMY on POD1 or POD3 were excluded from this study. Thus, all 1602 samples from 801 patients were successfully measured and analyzed (Table 1).

\section{Postoperative complications}

In total, postoperative complications, including minor complications, were diagnosed in 148 patients (18.5\%). Among them, 51 (6.4\%) were diagnosed as having CD grade III or higher POPF. Postoperative surveillance also revealed major complications diagnosed as CD grade III or higher, including anastomotic leakage in 19 patients $(2.3 \%)$, intra-abdominal bleeding in $14(1.7 \%)$, ileus in $5(0.6 \%)$ and pneumonia in $3(0.3 \%)$. POPFs were diagnosed and specific treatment, such as antibiotic administration and drain exchange, was started after a mean period of 7.3 POD (range, POD 1-34). The median duration of hospitalization was significantly prolonged in patients with grade III and higher POPFs, being 36.0 (range 10-91) days, compared with 10 (5-89) days $(P<0.001)$ for the patients without grade III or higher POPFs.

\section{Appropriate D-AMY cut-off levels and risk classification}

D-AMY levels grouped according to the presence of CD grade III or higher POPFs and timing of measurement are summarized in Fig. 1. D-AMY levels on POD1 in the patients who eventually developed severe POPF were significantly higher than those in patients without severe POPF 


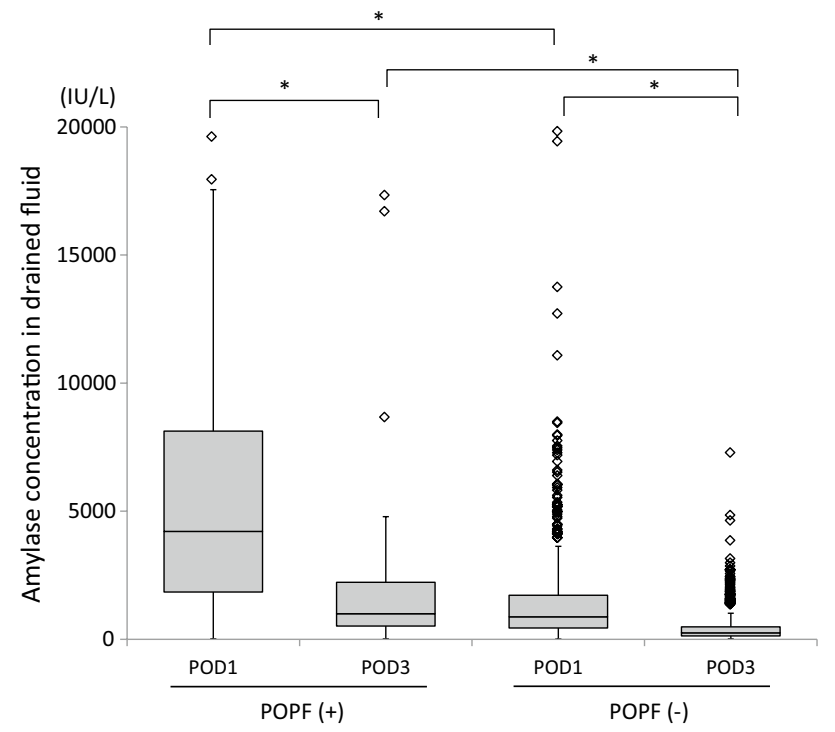

Fig. 1 Summary of D-AMY levels on POD1 and POD3 in patients with and without CD grade III or higher POPF using box-andwhisker plot and multiple comparison analysis by Steel-Dwass test $(* P<0.001)$

[4212 IU/L (range 177-19529 IU/L) vs 874.5 IU/L (range 34-19743 IU/L)] $(P \leq 0.001)$. Similarly, this difference was also found on POD3 [median of 995 IU/L (range 13-17216) vs $249.5 \mathrm{IU} / \mathrm{L}$ (range $11-7026 \mathrm{IU} / \mathrm{L})](P \leq 0.001)$. Timedependent changes between POD1 and POD3 were found

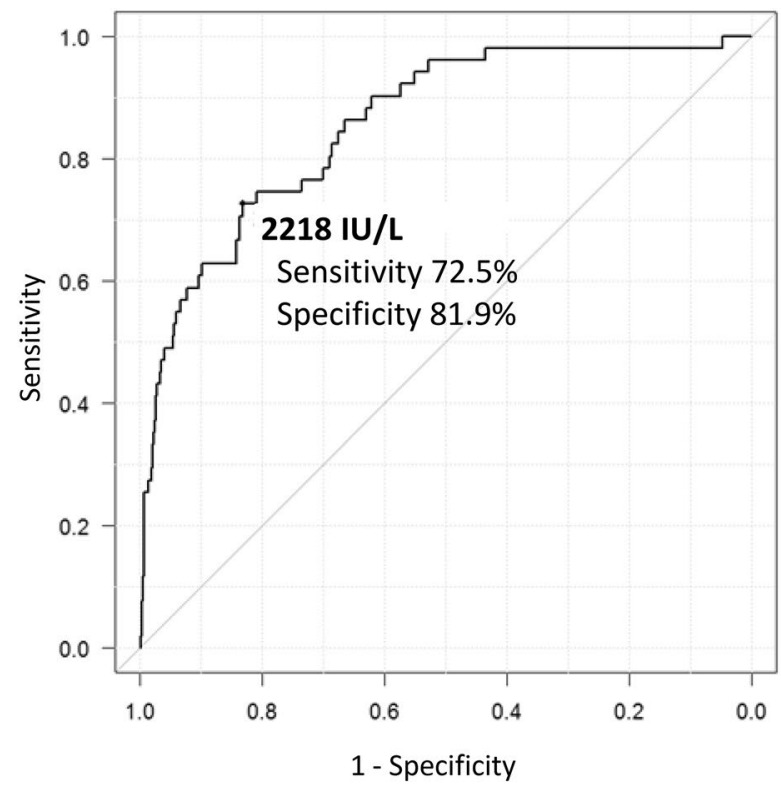

a ROC curve of D-AMY on POD1

Fig. 2 ROC curve showing the discriminative value of D-AMY on POD1 and POD3 for CD grade III or higher POPF. D-AMY levels on POD1 and POD3 exhibited an area under the curve of 0.855 and regardless of the presence of CD grade III or higher POPF $(P \leq 0.001)$.

Figure 2 shows the ROC curve for the discriminative value of D-AMY on POD1 and POD3 for CD grade III or higher POPFs. D-AMY on POD1 and POD3 exhibited an area under the curve of 0.855 and 0.818 , and the optimal cutoff values for prediction of CD grade III or higher POPFs were 2218 and $555 \mathrm{IU} / \mathrm{L}$, respectively.

Subjects were classified into four groups according to the D-AMY cutoffs, as shown in Table 2. This table shows the incidence of POPF grade III or higher in each group. It was obvious that the incidence of POPF was significantly higher in the high D-AMY group $\left(H_{\mathrm{POD} 1}, 22.6 \%\right)$ than in the low D-AMY group $\left(L_{\mathrm{POD} 1}, 2.2 \%\right)$ on POD1. The incidence in the $H_{\text {POD1 }}$ group was also significantly higher than for the subjects overall $(6.4 \%)$. This was also the case for POD3. Furthermore, the incidence of POPF in the $H_{\mathrm{POD} 1} H_{\mathrm{POD} 3}$ group, which had higher D-AMY levels than the cutoffs on both POD1 and POD3, was notably high (31.4\%), while the incidence in the $L_{\mathrm{POD} 1} L_{\mathrm{POD} 3}$ group, which had lower D-AMY levels than the cutoffs on both days, was only $1.7 \%$.

Table 3 shows the diagnostic ability of D-AMY when the cases were grouped according to the cut-off values. For comparison of diagnostic ability, we applied the likelihood ratio (LR) and diagnostic odds ratio (DOR), which are not affected by disease frequency and incidence of complications. Within single cut-off groups, positive LR [LR(+)] and DOR in the $H_{\text {POD1 }}$ group $(4.32,13.1)$ was higher than that in

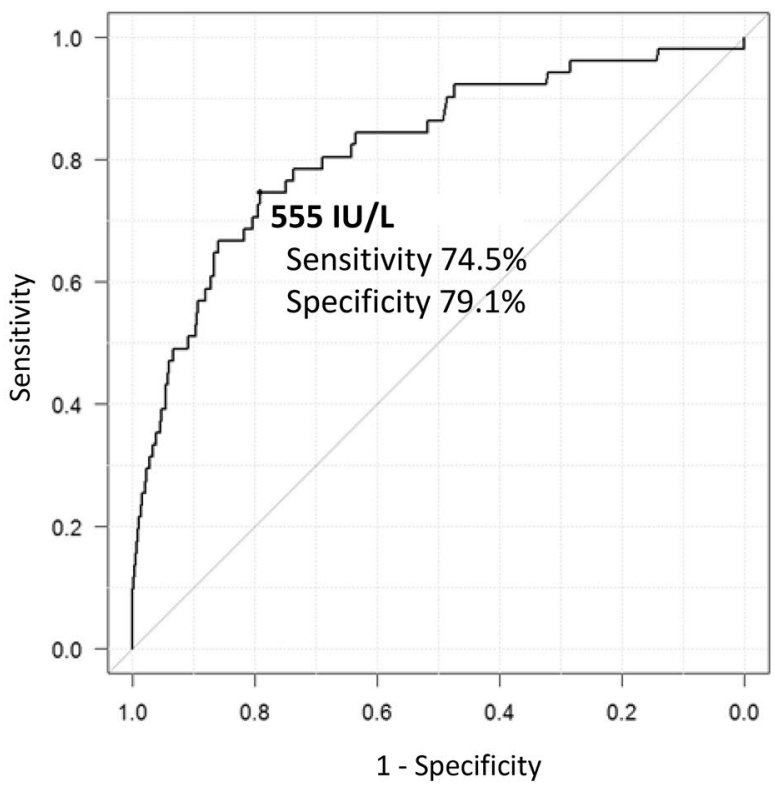

b ROC curve of D-AMY on POD3

0.818, and the optimal cut-off values for prediction of CD grade III or higher POPF were 2218 and 555 IU/L, respectively 
Table 2 Incidence of POPF in groups classified by the D-AMY cutoffs of POD1 and POD3

\begin{tabular}{lllr}
\hline & \multicolumn{2}{c}{ D-AMY on POD3 } & Total \\
\cline { 2 - 4 } & $>555 \mathrm{IU} / \mathrm{L}\left(H_{\mathrm{POD} 3}\right)$ & $<555 \mathrm{IU} / \mathrm{L}\left(L_{\mathrm{POD} 3}\right)$ & \\
\hline D-AMY on POD1 & & & \\
$>2218 \mathrm{IU} / \mathrm{L}\left(H_{\mathrm{POD} 1}\right)$ & $31.4 \%(33 / 105)$ & $6.9 \%(4 / 58)$ & $22.6 \%(37 / 163)$ \\
$<2218 \mathrm{IU} / \mathrm{L}\left(L_{\mathrm{POD} 1}\right)$ & $5.6 \%(5 / 90)$ & $1.7 \%(9 / 548)$ & $2.2 \%(14 / 638)$ \\
Total & $19.5 \%(38 / 195)$ & $1.8 \%(13 / 706)$ & $6.4 \%(51 / 801)$ \\
\hline
\end{tabular}

$D-A M Y$ amylase concentration of drained abdominal fluid, $P O D$ postoperative day, $H_{P O D I}$ the cases with D-AMY level higher than cutoff level in POD1, $H_{P O D 3}$ the cases with D-AMY higher than cutoff level in POD3

\begin{tabular}{lllllll}
\hline D-AMY (IU/L) & Sensitivity (\%) & Specificity $(\%)$ & Accuracy $(\%)$ & LR $(+)$ & LR (-) & DOR \\
\hline Single cutoff & & & & & & \\
$H_{\text {POD1 }}$ & 72.5 & 83.2 & 82.5 & 4.32 & 0.33 & 13.06 \\
$H_{\text {POD3 }}$ & 74.5 & 79.1 & 78.8 & 3.56 & 0.32 & 11.06 \\
Double cutoff & & & & & & \\
$H_{\text {POD1 }} H_{\text {POD3 }}$ & 64.7 & 90.4 & 88.8 & 6.74 & 0.39 & 17.26 \\
\hline
\end{tabular}

$D-A M Y$ amylase concentration of drained abdominal fluid, $L R(+)$ positive likelihood ratio, $L R(-)$ negative likelihood ratio, $D O R$ diagnostic odds ratio, $H_{P O D I}$ the cases with D-AMY level higher than cutoff level in POD1, $H_{P O D 3}$ the cases with D-AMY higher than cutoff level in POD3, $H_{P O D I} H_{P O D 3}$ the case with D-AMY levels higher than cutoff level both in POD1 and POD3, POD postoperative day
Table 3 Diagnostic accuracies of POPF in groups classified with D-AMY groups the $H_{\mathrm{POD} 3}$ group $(3.56,11.1)$, while there was no difference in negative $\mathrm{LR}[\mathrm{LR}(-)]$ (0.33 vs 0.32$)$. When double cutoffs were applied, $\mathrm{LR}(+)$ and DOR in the $H_{\mathrm{POD} 1} H_{\mathrm{POD} 3}$ group were notably high $(6.74,17.3)$.

\section{Risk factors for POPF}

The results of univariate and multivariate analyses of clinical factors such as sex, preoperative body mass index, surgical approach and procedure, operation time, intraoperative blood loss volume, and D-AMY for grade III and higher POPFs are summarized in Table 4. For blood loss volume and operation time, we used the medians as the cut-off levels. Univariate analysis confirmed statistical significance for all variables except the surgical approach. Multivariate analysis using logistic regression revealed that $H_{\mathrm{POD} 1} H_{\mathrm{POD} 3}$ D-AMY was an independent predictor with a considerably high odds ratio for the occurrence of grade III or higher POPF.

\section{Discussion}

In this retrospective cohort study, we hypothesized that a continuously high D-AMY level would clinically predict problematic POPF following gastrectomy. Therefore, we investigated the clinical applicability of measuring D-AMY at two points in the early postoperative period, i.e., POD1 and POD3. This approach, which incurs no extra cost and is simple and non-invasive to perform, has not been fully considered hitherto.

The 801 patients in the present study were classified into four groups on the basis of the two D-AMY cut-off values. The incidence of CD grade III or higher POPF in patients with higher D-AMY levels on both POD1 and POD3 was approximately five times higher than for the subjects as a whole, and nearly 20 times higher than the incidence in patients who had lower D-AMY levels on both POD1 and POD3. Multivariate analysis demonstrated that classification into the highest risk group was an independent risk factor associated with a notably high LR(+) and DOR for occurrence of severe POPF. These results highlighted the advantage of two-point D-AMY measurement, which improved the accuracy of POPF prediction by the early postoperative period, i.e., POD3.

One retrospective study of 76 selected cases has reported that patients, whose D-AMY level on POD3 was retained at $\geq 31.2 \%$ of that on POD1 were more likely to develop POPF [7]. Another study reported that the combination of $\mathrm{D}-\mathrm{AMY} \geq 2000 \mathrm{IU} / \mathrm{L}$ on POD1 and CRP $\geq 20 \mathrm{mg} / \mathrm{dL}$ on POD3 was predictive of CD grade III or higher POPF after laparoscopic distal gastrectomy [8]. These studies in fact suggested that multiple measurements over time would have clinical utility. In the present study, we addressed this issue with the aim of finding a simple and versatile diagnostic method for prediction of clinically problematic POPF in a large cohort of consecutive patients who underwent radical gastrectomy. 
Table 4 Univariate and multivariate analyses of associations between patient characteristics and grade III or higher postoperative pancreatic fistula according to ClavienDindo classification

\begin{tabular}{|c|c|c|c|c|}
\hline \multirow[t]{2}{*}{ Variables } & \multicolumn{2}{|l|}{ Univariate analysis } & \multicolumn{2}{|l|}{ Multivariate analysis } \\
\hline & POPF (grade III-) & $P$ value & $\begin{array}{l}\text { Odds ratio (95\% confi- } \\
\text { dence interval) }\end{array}$ & $P$ value \\
\hline Sex & & $<0.001$ & & 0.192 \\
\hline Male & $43(8 \%)$ & & $1.76(0.75-4.13)$ & \\
\hline Female & $8(3 \%)$ & & 1 & \\
\hline BMI $\left(\mathrm{kg} / \mathrm{m}^{2}\right)$ & & $<0.001$ & & 0.050 \\
\hline$>25$ & $23(13 \%)$ & & $1.93(1.00-3.72)$ & \\
\hline$<25$ & $28(5 \%)$ & & 1 & \\
\hline Approach & & 0.283 & & \\
\hline Open & $21(8 \%)$ & & & \\
\hline Laparoscopic & $30(6 \%)$ & & & \\
\hline Procedure & & 0.036 & & 0.407 \\
\hline TG & $15(10 \%)$ & & $1.44(0.61-3.45)$ & \\
\hline DG/PG/PPG & $36(5 \%)$ & & 1 & \\
\hline Splenectomy & & 0.024 & & 0.948 \\
\hline Yes & $5(18.5 \%)$ & & $0.96(0.24-3.78)$ & \\
\hline No & $46(5.9 \%)$ & & 1 & \\
\hline Operation duration $(\mathrm{min})$ & & 0.020 & & 0.130 \\
\hline$>295$ & $34(8 \%)$ & & $1.70(0.88-3.40)$ & \\
\hline$<295$ & $17(4 \%)$ & & 1 & \\
\hline Blood loss (g) & & 0.009 & & 0.094 \\
\hline$>70$ & $35(9 \%)$ & & $1.89(0.90-4.00)$ & \\
\hline$<70$ & $16(4 \%)$ & & 1 & \\
\hline D-AMY group & & $<0.001$ & & $<0.001$ \\
\hline$H_{\mathrm{POD} 1} H_{\mathrm{POD} 3}$ & $33(31 \%)$ & & $15.2(7.83-29.6)$ & \\
\hline Other* & $18(3 \%)$ & & 1 & \\
\hline
\end{tabular}

$B M I$ body mass index, $C I$ confidence interval, $D-A M Y$ amylase concentration of drained abdominal fluid, $D G$ distal gastrectomy, $H_{P O D I} H_{P O D 3}$ the case with D-AMY levels higher than cutoff level both in POD1 and POD3, $P G$ proximal gastrectomy, $P O D$ postoperative day, $P O P F$ postoperative pancreatic fistula, $P P G$ pylorus preserving gastrectomy, $T G$ total gastrectomy

*The case other than patients with higher D-AMY than both cutoffs in POD1 and POD3
For patients who are considered to be in the highest risk group, it is necessary to prevent or control the development of severe POPF, for instance, by administration of selective antibiotics and proactive drainage optimization. With regard to the use of antibiotics, prolonged prophylactic antibiotics generally does not benefit for postoperative patients [19, 20]. On the other hand, several studies have reported that prophylactic administration of antibiotics for acute pancreatitis is associated with a significantly lower incidence of pancreatic or peripancreatic infections, mortality and sepsis. Therapeutic administration of antibiotics is recommended as early as possible after diagnosis of infectious POPF [21, 22]. Preliminary additional prophylactic antibiotic administration is likely to be beneficial for preventing deterioration in particular patients with a high risk of POPF and subsequent intra-abdominal abscess formation. Although so far, the indications for antibiotic administration and the patients who would benefit from such treatment for acute pancreatitis have remained unclear [23], risk classification as employed in the present study might help to indicate candidates who are suitable for preliminary antibiotic administration according to their individual risk of POPF.

With regard to drainage, routine prolonged placement of an indwelling drain can be potentially harmful, and any unnecessary drains should be removed as soon as possible to prevent retrograde infections [24-26]. However, appropriate drainage remains the principal treatment for intra-abdominal abscess.

From the results of the present study, we propose one possible stepwise strategy for postoperative management. For patients considered at risk of progression to severe POPF on the basis of intraoperative findings or clinical condition, prophylactic drain tubes should be inserted and D-AMY should be measured on POD1. If the D-AMY level is high, the drain should be left until POD3 when D-AMY is measured again. Despite the substantially lower incidence of severe POPF in patients who had a low D-AMY level on POD3, those with higher D-AMY levels on both POD1 and POD3 had 
a considerably high POPF occurrence rate. Therefore, for the highest risk group in our study, leaving the drain tube in place might be meaningful for monitoring and intervention on demand. For patients in the highest risk group, careful management, such as taking cultures of drained fluid for antibiotic optimization and early visualization of the intraabdominal condition by CT scan or X-ray contrast examination, as well as adjustment of the drain position, would be inevitable. On the other hand, for patients with a low risk of POPF as indicated by the D-AMY levels, other than $H_{\mathrm{POD} 1} H_{\mathrm{POD} 3}$ group, the prophylactic drain tube might perhaps be removed earlier. Furthermore, in the $L_{\mathrm{POD} 1} L_{\mathrm{POD} 3}$ cases, the drain might be removed more safely. Two-point D-AMY measurement has the advantage of extracting highrisk as well as low-risk patients by POD3.

The present study had some limitations, including the retrospective determination of the cut-off values. In addition, the volume of drainage fluid, which affects the concentration of D-AMY through dilution, especially in case of massive lymphorrhea, was not taken into consideration. However, this exploratory study offered insight into the applicability of a simple and useful diagnostic tool. It will be necessary to evaluate the clinical significance of risk-based treatment in a future prospective study.

In summary, this study has demonstrated the clinical significance of two-point measurement of the D-AMY level in drainage fluid on POD1 and POD3 in a large sample cohort. This two-point D-AMY measurement method is simple to perform and can predict the incidence of POPF, possibly allowing appropriate management of patients by classifying them according to individual risk. A prospective study should be conducted to verify the applicability of individualized management based on POPF risk.

\section{Compliance with ethical standards}

Conflict of interest The authors have no conflicts of interest to report.

Ethical standards All procedures followed were in accordance with the ethical standards of the institutional and national committees responsible for human experimentation, and with the 1964 and later versions of the Helsinki Declaration. Informed consent for inclusion in the study was obtained from all patients or their representatives.

\section{References}

1. Sano T, Sasako M, Katai H, Maruyama K. Amylase concentration of drainage fluid after total gastrectomy. Br J Surg. 1997;84:1310-2.

2. Miki Y, Tokunaga M, Bando E, Tanizawa Y, Kawamura T, Terashima M. Evaluation of postoperative pancreatic fistula after total gastrectomy with D2 lymphadenectomy by ISGPF classification. J Gastrointest Surg. 2011;15:1969-76.
3. Iwata N, Kodera Y, Eguchi T, Ohashi N, Nakayama G, Koike $\mathrm{M}$, et al. Amylase concentration of the drainage fluid as a risk factor for intra-abdominal abscess following gastrectomy for gastric cancer. World J Surg. 2010;34:1534-9.

4. Tomimaru Y, Miyashiro I, Kishi K, Motoori M, Yano M, Shingai $\mathrm{T}$, et al. Is routine measurement of amylase concentration in drainage fluid necessary after total gastrectomy for gastric cancer? J Surg Oncol. 2011;104:274-7.

5. De Sol A, Cirocchi R, Di Patrizi MS, Boccolini A, Barillaro I, Cacurri A, et al. The measurement of amylase in drain fluid for the detection of pancreatic fistula after gastric cancer surgery: an interim analysis. World J Surg Oncol. 2015;19(13):65.

6. Obama K, Okabe H, Hosogi H, Tanaka E, Itami A, Sakai Y. Feasibility of laparoscopic gastrectomy with radical lymph node dissection for gastric cancer: from a viewpoint of pancreasrelated complications. Surgery. 2011;149:15-21.

7. Kanda M, Fujiwara M, Tanaka C, Kobayashi D, Iwata N, Mizuno A, et al. Predictive value of drain amylase content for peripancreatic inflammatory fluid collections after laparoscopic (assisted) distal gastrectomy. Surg Endosc. 2016;30:4353-62.

8. Kobayashi D, Iwata N, Tanaka C, Kanda M, Yamada S, Nakayama $\mathrm{G}$, et al. Factors related to occurrence and aggravation of pancreatic fistula after radical gastrectomy for gastric cancer. J Surg Oncol. 2015;112:381-6.

9. Jiang X, Hiki N, Nunobe S, Kumagai K, Nohara K, Sano T, et al. Postoperative pancreatic fistula and the risk factors of laparoscopy-assisted distal gastrectomy for early gastric cancer. Ann Surg Oncol. 2012;19:115-21.

10. Lo CH, Chen JH, Wu CW, Lo SS, Hsieh MC, Lui WY. Risk factors and management of intra-abdominal infection after extended radical gastrectomy. Am J Surg. 2008;196:741-5.

11. Nobuoka D, Gotohda N, Konishi M, Nakagohri T, Takahashi S, Kinoshita T. Prevention of postoperative pancreatic fistula after total gastrectomy. World J Surg. 2008;32:2261-6.

12. Aranha GV, Aaron JM, Shoup M, Pickleman J. Current management of pancreatic fistula after pancreaticoduodenectomy. Surgery. 2006;140:561-8.

13. Japanese Gastric Cancer Association. Japanese classification of gastric carcinoma: 3rd English edition. Gastric Cancer. 2011;14(2):101-12.

14. International Union Against Cancer. TNM classification of malignant tumours. 7th ed. Chichester: Wiley; 2009.

15. Japanese Gastric Cancer Association. Japanese gastric cancer treatment guidelines 2014 (ver. 4). Gastric Cancer. 2017;20(1):1-19.

16. Bassi C, Dervenis C, Butturini G, Fingerhut A, Yeo C, Izbicki J, International Study Group on Pancreatic Fistula Definition, et al. Postoperative pancreatic fistula: an international study group (ISGPF) definition. Surgery. 2005;138:8-13.

17. Kung CH, Lindblad M, Nilsson M, Rouvelas I, Kumagai K, Lundell L, et al. Postoperative pancreatic fistula formation according to ISGPF criteria after D2 gastrectomy in Western patients. Gastric Cancer. 2014;17:571-7.

18. Clavien PA, Barkun J, de Oliveira ML, Vauthey JN, Dindo $\mathrm{D}$, Schulick RD, et al. The Clavien-indo classification of surgical complications: five-year experience. Ann Surg. 2009;250:187-96.

19. Bratzler DW, Houck PM, Surgical Infection Prevention Guideline Writers Workgroup. Antimicrobial prophylaxis for surgery: an advisory statement from the National Surgical Infection Prevention Project. Am J Surg. 2005;189:395-404.

20. Imamura H, Kurokawa Y, Tsujinaka T, Inoue K, Kimura Y, Iijima S, Shimokawa T, Furukawa H. Intraoperative versus extended antimicrobial prophylaxis after gastric cancer surgery: a phase 3, open-label, randomised controlled, non-inferiority trial. Lancet Infect Dis. 2012;12:381-7. 
21. Fritz S, Hartwig W, Lehmann R, Will-Schweiger K, Kommerell M, Hackert T, Schneider L, Büchler MW, Werner J. Prophylactic antibiotic treatment is superior to therapy on-demand in experimental necrotising pancreatitis. Crit Care. 2008;12(6):R141.

22. Xu T, Cai Q. Prophylactic antibiotic treatment in acute necrotizing pancreatitis: results from a meta-analysis. Scand J Gastroenterol. 2008;43(10):1249-58.

23. Mourad MM, Evans R, Kalidindi V, Navaratnam R, Dvorkin L, Bramhall SR. Prophylactic antibiotics in acute pancreatitis: endless debate. Ann R Coll Surg Engl. 2017;99(2):107-12.

24. Mangram AJ, Horan TC, Pearson ML, Silver LC, Jarvis WR, Guideline for prevention of surgical site infection. Hospital infection control practices advisory committee. Infect Control Hosp Epidemiol. 1999;1999(20):250-78.
25. Kawai M, Tani M, Terasawa H, Ina S, Hirono S, Nishioka R, et al. Early removal of prophylactic drains reduces the risk of intra-abdominal infections in patients with pancreatic head resection: prospective study for 104 consecutive patients. Ann Surg. 2006;244:1-7.

26. Petrowsky H, Demartines N, Rousson V, Clavien PA. Evidence-based value of prophylactic drainage in gastrointestinal surgery: a systematic review and meta-analyses. Ann Surg. 2004;240:1074-84. 